\title{
Dissolving the Measurement Problem Is Not an Option for the Realist
}

\author{
Matthias Egg \\ University of Bern, Institut für Philosophie, Länggassstrasse 49, CH-3012 Bern, Switzerland
}

\begin{abstract}
This paper critically assesses the proposal that scientific realists do not need to search for a solution of the measurement problem in quantum mechanics, but should instead dismiss the problem as ill-posed. James Ladyman and Don Ross have sought to support this proposal with arguments drawn from their naturalized metaphysics and from a Bohr-inspired approach to quantum mechanics. I show that the first class of arguments is unsuccessful, because formulating the measurement problem does not depend on the metaphysical commitments which are undermined by ontic structural realism, rainforest realism, or naturalism in general. The second class of arguments is problematic due to its refusal to provide an analysis of the term "measurement". It turns out that the proposed dissolution of the measurement problem is in conflict not only with traditional forms of scientific realism but even with the rather minimal realism that Ladyman and Ross themselves defend. The paper concludes with a brief discussion of two related proposals: Healey's pragmatist approach and Bub's information-theoretic interpretation.
\end{abstract}

\section{Introduction}

One of the attractions of non-realist approaches to quantum mechanics (QM) is that they offer a way to dissolve the measurement problem instead of adopting a solution for it that would either have to modify the physics (such as theories with additional variables or spontaneous collapses) or drastically inflate the empirically inaccessible content of reality (such as many-worlds interpretations). Nonetheless, many metaphysicians (and some physicists) consider the abandonment of realism too high a price to pay and therefore insist that the measurement problem calls for a (realistic) solution rather than a dissolution along non-realist lines.

Could there be a third position that somehow combines the attractions of these two (seemingly opposing) camps? In this paper, I critically assess a recent proposal for such a position, which can be found in the writings of James

Email address: matthias.egg@philo.unibe.ch (Matthias Egg) 
Ladyman and Don Ross (2007, 2013). While describing their approach to the measurement problem as "roughly the sort of account favoured by earlier versions of the Copenhagen interpretation" (2013, 134), Ladyman and Ross do not think this amounts to instrumentalism, but rather seek to defend it as part of their brand of scientific realism, which combines ontic structural realism (OSR) with what they call "rainforest realism" (as developed in Chapters 3 and 4, respectively, of their seminal (2007) book Every Thing Must Go). The purpose of the present paper is to show that their Copenhagen-style dissolution of the measurement problem does not fit well with a position that presents itself as a version of scientific realism.

In order to avoid misunderstandings, let me first mention an important point of agreement between Ladyman/Ross and myself. Much of their (2013) argument is directed against the simple realism-versus-instrumentalism dichotomy (with respect to QM), which they find operative in the philosophy of David Deutsch (2011). More specifically, they argue that there is much within the formalism of QM about which one can be a realist despite not being committed to any realistic solution of the measurement problem. I largely agree with this claim, noting that one need not be an ontic structural realist to appreciate this point (cf. Cordero 2001; Saatsi forthcoming). Nor am I particularly worried about Michael Esfeld's (2013) diagnosis of OSR being only a partial realism if it does not incorporate a realist treatment of the measurement problem 11 What I do criticize is that the dissolution of the measurement problem proposed by Ladyman and Ross undermines some specific commitments that should be part of any position deserving to be called realism (even only a partial one).

My investigation proceeds as follows. In Section 2 I will consider how the different elements of naturalized metaphysics as developed by Ladyman and Ross might seem to undermine the traditional formulation of the measurement problem, insofar as the latter depends on applying the quantum formalism to macroscopic objects. My contention will be that while naturalized metaphysics incorporates some telling arguments against traditional views of how the macroscopic realm relates to the microworld, none of them justifies viewing the measurement problem as a pseudo-problem. Section 3 will then show how Ladyman's and Ross's Bohr-inspired alternative approach to the measurement problem contradicts some widely shared realist (and, relatedly, naturalist) commitments. This by itself may not be so problematic for Ladyman and Ross, because they do not subscribe to standard realism anyway. I will therefore continue my argument in Section 4 by demonstrating that some of Ladyman's and Ross's own arguments in favor of scientific realism are in tension with their proposed dissolution of the measurement problem. The concluding Section 5 will briefly look at two related proposals and will give reasons against thinking that these have a better prospect of being compatible with realism than the original one.

\footnotetext{
${ }^{1}$ In fact, I endorse a version of partial realism myself (Egg 2014).
} 


\section{Quantum states of macroscopic objects}

A key step in setting up the measurement problem consists in assigning a quantum state to a measurement apparatus. In the standard example (see, e.g., Myrvold 2017, Section 4), two possible final states of the apparatus are denoted by |"0" $\rangle_{A}$ and $|" 1 "\rangle_{A}$, corresponding to the two basis states $|0\rangle_{S}$ and $|1\rangle_{S}$ of the measured quantum system. The measurement problem then consists in making sense of the superposed final state $a|0\rangle_{S}|" 0 "\rangle_{A}+b|1\rangle_{S}|" 1 "\rangle_{A}$, which, if $a$ and $b$ are both nonzero, seems to contradict our experience that instrument readings for such an experiment are always either "0" or " 1 ".

The assumption that the quantum formalism is the appropriate tool for describing not only microscopic entities like electrons but also macroscopic objects like measurement devices is rather widespread, so it may be surprising to see Ladyman and Ross question it. Let us therefore have a close look at their reasons for doing so:

Note that the way we set up the measurement problem relies on the idea that the state of an apparatus for measuring, say, spin in the $\mathrm{x}$-direction, is a quantum state that can be represented in the usual way by a ket vector $\mid$ reads 'up' $\rangle$. The usual rationale for treating this as a quantum state is that the apparatus is supposed to be made of a very large number of quantum particles, but nonetheless is still essentially the same kind of thing as the electron it is measuring. However, on the view of higher-order ontology sketched above (and explained in detail in 2007, Chapter 4]), there is no reason to regard the measuring device as something that exists at all from a microscopic perspective. We have also made clear our hostility to the idea that macroscopic objects are fundamentally made of microscopic ones. Hence, the application of the quantum formalism to macroscopic objects is not necessarily justified,... (Ladyman and Ross 2007, 182; quotation to be continued in Subsection 2.3 below)

This passage shows how the two elements of Ladyman's and Ross's realism motivate their reservations about extending the quantum description from the microscopic to the macroscopic domain: While OSR denies that "macroscopic objects are fundamentally made of microscopic ones", rainforest realism proposes a view of higher-order ontology that tells against using the same kinds of description for structures at different scales. I will now discuss these two elements and then turn to a third element which shifts the focus from realism to naturalism.

\subsection{Composition, OSR, and the measurement problem}

What is it, exactly, that prompts Ladyman and Ross (2007, 182) to "deny that measurement devices are the mereological sums of quantum particles"?

A first reason is their dissatisfaction with traditional philosophical accounts of mereology and composition. Early on in Every Thing Must Go, they describe 
how analytic metaphysicians have been misled by their hankering after a general a priori notion of composition, which pays little or no attention to what we have learnt about specific composition relations described within various sciences. In conclusion, they note: "We have no reason to believe that an abstract composition relation is anything other than an entrenched philosophical fetish" (2007, 21).

However, this kind of criticism can be dealt with rather quickly for our purpose, by noting that no abstract metaphysical composition relation is presupposed by the claim that measurement devices are composed of quantum systems. Instead, QM itself furnishes us with the rules of how systems combine to form larger systems, and it is those rules that tell us that the compound systems can be in superposed states just as the elementary systems can. Furthermore, insofar as the quantum formalism describes interactions between the parts of compound systems, the quantum mechanical composition relation also has the necessary dynamical character that Ladyman (2017, 156) finds missing in the traditional metaphysical accounts of composition.

In the same context, Ladyman mentions the renormalization group to illustrate the vast difference between the naïve metaphysical picture of composition and the intricate way in which actual condensed matter physics describes how gross matter behaves in terms of interactions between atoms, electrons, and fields. Again, the reference to scientific accounts of composition is well taken, but I do not see how it could undermine the idea that matter is composed of quantum particles. In an important sense, this latter idea provides the very motivation for applying renormalization group methods in condensed matter physics, as a tool to eliminate degrees of freedom associated with the atomic constitution of matter that are irrelevant for its macroscopic behavior. At the same time, quantum measurement devices are characterized by the fact that some microscopic degrees of freedom (namely the ones being measured) are not eliminated, but do indeed affect the device's macroscopic behavior. The renormalization group has nothing to say about these degrees of freedom and it therefore does not tell against describing the measurement device with respect to them in the way that gives rise to the measurement problem.

A further possible reason to reject the idea of macroscopic objects being composed of quantum particles is the radical difference between the way in which quantum theories describe particles and our pre-theoretic notion of particles as "little things". Recognition of this difference lies at the heart of OSR, and it is summarized by Ladyman and Ross $(2013,143)$ as follows:

Quantum entanglement in particular and quantum physics in general, especially quantum field theory, show that there is no sense at all in which atoms or sub-atomic particles resemble little macroscopic things reduced drastically in size. In undomesticated physics, particles don't resemble any kind of entity that people had ever imagined prior to the twentieth century.

One might criticize these statements as exaggerated (for example, by referring to some experimental procedures in nanotechnology, which allow us to 
manipulate atoms in ways that are not so different from how we manipulate more familiar things), but that is not the point here. The question is, rather, whether any of the surprising features of quantum particles do indeed exclude the view that measuring devices are composed of them, or in other words, whether OSR does indeed undermine the usual way of setting up the measurement problem.

The historically most important and most widely discussed issue in OSR's account of particles concerns their identity and individuality (or lack thereof; see Ladyman 2016 and references therein). This issue, however, does not seem to have much relevance for the pertinence of viewing macroscopic objects as composed of quantum systems. On the contrary, the fact that there is a metaphysical debate on the (non-)individuality of quantum particles at all precisely shows that the quantum mechanics of composite systems is to some extent insensitive to whether the components are regarded as individuals or not. It is only their cardinality that matters, and this latter is unproblematic in non-relativistic QM (I will turn to relativistic quantum theory in the next subsection). Another way to make the same point is to note that the formalism of quantum mechanics already incorporates the features that fueled the debate on identity and individuality (namely, the indistinguishability postulate in quantum statistics and the non-supervenience of entanglement relations), so one should not expect the results of that debate to undermine the quantum mechanical account of composition.

Neither is it relevant whether particles are regarded as elementary (or fundamental) in any strong sense. I fully agree with Ladyman $(2016,202)$ that we should not regard them in this way, but nothing in the usual formulation of the measurement problem depends on doing so. In fact, there is excellent empirical evidence for the occurrence of superposed states in unambiguously non-elementary quantum systems (see Arndt and Hornberger 2014 for a recent review), so the whole problem can be set up without any reference to elementary or fundamental particles.

Correspondingly, admitting the measurement problem as a real problem does not presuppose taking QM as the final word on the fundamental ontology of the world. Future physics may indeed present us with fundamental theories of a completely different structure, but whatever these theories look like, they will have to accommodate those basic features of QM which allow us to predict the well-confirmed interference phenomena that motivated the development of QM in the first place. Quantum superposition is one of these features, and none of the arguments discussed so far gives us any reason to suppose that it disappears when many quantum systems combine (according to the rules of QM itself) to form a macroscopic apparatus.

\subsection{Rainforest realism: particles and measurement devices as real patterns}

While OSR is mostly concerned with what particles are not (namely, fundamental and self-subsistent individuals), rainforest realism tells us what they are: just like other elements of higher-order ontology, particles are real patterns (Ladyman 2016, 203-204). The notion of a real pattern is adopted from Dennett (1991) and discussed in detail in Ladyman and Ross (2007. Chapter 4), but 
for present purposes, the following elucidation (due to Wallace 2003, 93) will suffice: "the existence of a pattern as a real thing depends on the usefulness - in particular, the explanatory power and predictive reliability - of theories which admit that pattern in their ontology".

Recognizing particles as real patterns (rather than fundamental entities) is important, because the particle concept becomes increasingly problematic when we turn from non-relativistic quantum mechanics to relativistic quantum field theory. The appearance of particle creation and annihilation not only undermines the above-mentioned appeal to a well-defined cardinality of particles in a composite system, but it also blurs the line between particles as persisting objects and mere excitations of quantum fields. More precisely, this latter distinction now becomes dependent on the time and energy scale at which a system is considered (Ladyman 2017, 158). The merit of rainforest realism is that it takes this dependence into account and makes room for scale-relative ontological commitments.

This implies that reference to particles is unproblematic as long as the context is appropriately specified in terms of the relevant time and energy scale. Formulations of the measurement problem implicitly do this by involving only two kinds of physical systems, namely non-relativistic quantum particles and macroscopic measurement devices. Neither of them requires consideration of quantum field theoretic effects, hence the scale at which the measurement problem is formulated is not affected by the breakdown of the particle concept in quantum field theory.

Now the notion of real patterns does not only apply to particles but also to measurement devices, and this opens another way to criticize the setting up of the measurement problem. Continuing a statement already quoted above, Ladyman and Ross (2007, 182-183) write:

In sum, then, we deny that measurement devices are the mereological sums of quantum particles. Rather, they are real patterns and their states are legitimate posits of science in so far as they enable us to keep track of the phenomena. They do not enable us to do this if we regard them as quantum states, and therefore so regarding them is not warranted.

The idea here seems to be that QM has a more limited domain of application than supposed by those who view measurement devices as composed of quantum particles: there is an autonomous science of the macroworld in the sense that QM simply doesn't apply to macroscopic measurement devices. ${ }^{2}$

However, some care is needed in specifying what exactly is meant by this supposed autonomy of the macroworld. Obviously, one cannot completely disregard QM when describing the states of macroscopic measurement devices. Reiterating a point made above in the context of renormalization: the very

\footnotetext{
${ }^{2}$ I owe this suggestion to an anonymous referee.
} 
point of performing a quantum measurement is the realization that the macroscopic domain is not autonomous, but influenced by some microscopic degrees of freedom (the ones being measured). Ladyman and Ross are, of course, aware of this, and they propose the Born rule as a sufficient description of how the quantum domain affects the macroscopic domain. This proposal will be discussed in Section 3. For the moment, let us just note that rainforest realism (and in particular, the idea of scale-relative ontology) should not be taken to imply an unqualified autonomy of the macroscopic scale.

\subsection{Naturalism and the assignment of quantum states}

The arguments discussed so far in this section were mainly based on theoretical or even metaphysical considerations. We now turn to a more empirically oriented line of argument that involves Ladyman's and Ross's commitment to naturalism. The following passage is a direct continuation of the one quoted at the beginning of this section:

Hence, the application of the quantum formalism to macroscopic objects is not necessarily justified, especially if those objects are importantly different from microscopic objects, as indeed they are, in not being carefully isolated from the environment. From the point of view of the [Principle of Naturalistic Closure], the representation of macroscopic objects using quantum states can only be justified on the basis of its explanatory and predictive power and it has neither. In fact, $\mathrm{QM}$ is explanator[il]y and predictively inaccurate at this scale since it entails that there ought to be superpositions that are not in fact observed. The predictive success of QM in this context consists in the successful application of the Born rule, and that is bought at the cost of a pragmatic splitting of the world into system and apparatus. (Ladyman and Ross 2007, 182)

The suggestion that a quantum description becomes inappropriate to the extent that systems fail to be isolated has some initial plausibility, as the interference effects indicating quantum behavior are indeed only observed for systems that are sufficiently well isolated from their environment. It is, however, questionable whether this serves as a general argument against applying the quantum formalism to macroscopic, non-isolated objects. First, one might ask whether QM is not required to explain various effects in such objects, ranging from superconductivity and superfluidity to such everyday phenomena as semiconductivity or ferromagnetism. I will not dwell on these examples because they would require a rather technical analysis of whether the explanatory and predictive success of QM in each case really depends on representing macroscopic objects using quantum states or (as in the case of measurement) on the successful application of some pragmatic recipe such as the Born rule. And even if it could be shown that the former is the case, Ladyman and Ross could still reply that the measurement case differs relevantly from the other cases, due to 
the manifest explanatory and predictive inadequacy of applying the quantum formalism to the measurement device.

What is needed, therefore, is some justification for assigning quantum states to a sufficiently general class of macroscopic objects that includes measurement devices. Obviously, such justification cannot be directly empirical, because there is indeed a sense in which QM (without some kind of projection postulate) "entails that there ought to be superpositions that are not in fact observed" 3 There is, however, a kind of indirect empirical justification that should also be acceptable to the naturalist and that becomes apparent as soon as we inquire into how the lack of isolation influences the behavior of quantum systems. Our present most successful treatment of this issue is through the theory of decoherence (see Bacciagaluppi 2016 for a review). By operating entirely within the formalism of QM, this theory presupposes just what Ladyman and Ross seek to prohibit: the assignment of quantum states to macroscopic objects (the environment) 4 Such assignments are therefore not just a philosopher's fancy, but are soundly rooted in scientific theorizing.

Ladyman and Ross (2007, 176-177) discuss decoherence theory in the context of what they call "Everett-Saunders-Wallace quantum mechanics" (a version of the many-worlds interpretation). They are somewhat sympathetic to that interpretation, but do not endorse it, because they are "not yet convinced that an Everettian plurality is the most consilient way of looking at contemporary physics" (182). So if decoherence theory only played a role in the Everettian approach to QM, Ladyman and Ross could legitimately disregard it and its practice of assigning quantum states to macroscopic objects. However, decoherence theory is by no means confined to an Everettian approach; its importance extends to all the major approaches to QM (Bacciagaluppi 2016. Section 3), and some of its central results are arguably interpretation-independent (Rosaler 2016). This is the kind of scientific theory that a naturalist should take ontologically seriously, which, in this case, means to accept the legitimacy of applying the quantum formalism to macroscopic objects.

\section{Leaving "measurement" unanalyzed?}

Having discussed Ladyman's and Ross's view on how not to apply QM to the macroscopic realm, let me now turn to the more positive part of their proposal. It starts with the remark (already quoted in Subsection 2.3) that the success of QM in a measurement context consists in the successful application of the Born

\footnotetext{
3 This should not be taken to suggest that we could experimentally test for interference between the terms of a macroscopic superposition and thereby falsify a prediction of QM. What is meant here by the failure to "observe superpositions" is merely the fact that, for example, we never seem to observe a measuring device displaying two different results of a single measurement at once.

${ }^{4} \mathrm{As}$ is well known, the fact that decoherence theory is just an application of standard QM also implies that decoherence by itself does not solve the measurement problem (Bacciagaluppi 2016, Section 2.1).
} 
rule. This is indeed successful if we simply insist (as Niels Bohr famously did) that the apparatus needs to be described classically in the sense of not being in any superposed state. Ladyman and Ross $(2013,134)$ explicitly sympathize with Bohr's early version of the Copenhagen interpretation, which they view as distinct from later versions in virtue of its refusal to give any story about collapse of the wave function.

Without entering into the complex debate on the history of the Copenhagen interpretation, it is noteworthy that Ladyman and Ross (ibid.) identify "an abandonment not so much of realism as of naturalism itself" in the transition from Bohr to later versions of Copenhagen, which "did include a story about collapse, but interpreted it as a consequence of measurement". Against this assessment, I submit that the abandonment of naturalism (and realism) takes place when one endorses Ladyman's and Ross's reading of early Copenhagen, not when one switches from this version to a later one.

Ladyman's and Ross's argument for viewing Bohr's approach as compatible with scientific realism depends on the interpretation-independent content of standard QM already mentioned in Section 1], where I admitted that one can be a realist about large parts of $\mathrm{QM}$ without committing to any particular solution of the measurement problem. However, as we just saw, some of that content gets its empirical character only via the successful application of the Born rule - the content is interpretation-independent precisely because any viable interpretation of $\mathrm{QM}$ needs to incorporate the Born rule in some way.

Now the problem with the Born rule is that it speaks about the probabilities of measurement results, while it is notoriously unclear what counts as a "measurement". This critique is well known, and it is often put in terms of awkward questions for those who (in the spirit of later Copenhagen) tie the notion of measurement to a collapse of the wave function. So for example, John S. Bell (1990, 19) famously asked whether a single-celled living creature already qualified to play the role of "measurer" or whether it takes some better qualified system (with a Ph.D.?) to make the wave function collapse. But the basic point of criticism (as Bell makes clear in the rest of his paper) does not depend on any specific view of wave function collapse, but on using such a desperately imprecise notion as "measurement" in a basic assumption of physics. This is why realistic versions of QM (such as those associated with the names of Everett, Bohm, or GRW) seek to derive the Born rule by giving a physical account of what it is to be a measurement. Bohr, on the other hand, denies the need for such an account, as Ladyman and Ross $(2013,134)$ point out approvingly.

Admittedly, any theory has to operate with some basic notions which are not amenable to further analysis, so why not simply treat "measurement" as such a notion? This works well for situations in which we all agree whether the notion applies or not. But what about ambiguous cases, for example, a device that displays a measurement outcome which is not (even indirectly) observed ${ }^{5}$ by

${ }^{5}$ A useful explication of the notion of "observation" relevant for this context is given by Ladyman and Ross (2007 307) in terms of "informational connectedness". In the following, I have 
anyone? Bohr repeatedly insisted that human observers play no essential role in the measurement process, but how can this be justified without an analysis of "measurement"? Neither our pre-theoretical nor our scientific usage of the word "measurement" seems to settle the question whether unobserved measurements should still count as measurements.

There are two possible lines of response to this problem, both of which are to some extent explored by Ladyman and Ross. First, one might argue that even in the absence of a physical analysis of "measurement", the notion can be made sufficiently precise in information-theoretic terms. I will briefly comment on this idea in Subsection 5.2 below. The second option is to go verificationist and to denounce any question about unobserved measurements as a pseudo-question: Such events (by definition) do not make any difference to what we observe, hence we should not suppose that there are any matters of fact concerning them. However, this is hard to square with realism, understood as a stance that refuses to limit reality to what we can observe, or worse still, to what we actually do observe. Ladyman and Ross (2007, 309) are quite honest about how their verificationism limits the domain of what counts as real, but the conflict with realism is obscured by the somewhat far-fetched example they give in that context: Most realists will readily agree that "there are no grounds for regarding the other side of [the Big Bang] as part of reality" (ibid.). By contrast, many realists will think that something has gone deeply wrong if we are discouraged from believing that there is a fact of the matter as to how our measurement devices behave when no one watches them.

Before I turn (in the next section) to the question in how far Ladyman's and Ross's own version of realism should be bothered by this tension, I should also mention that there is something anti-naturalistic about drawing such anthropocentric limits around what counts as real. A thorough discussion of Ladyman's and Ross's (2007, Section 6.3) arguments for the compatibility of naturalism and verificationism is beyond the scope of this paper. Suffice it to say that these arguments are most plausible when the verifiability criterion is understood epistemically (as a policy on what our theorizing should or should not be concerned with) rather than metaphysically (as a criterion on what does or does not belong to reality). To the extent that the latter reading is implied, a naturalist is likely to wonder why reality should care which parts of it are accessible to our observation.

\section{Unobserved measurements and objective modality}

That the Bohrian approach to the measurement problem entails a conflict with some widespread realistic and naturalistic intuitions may not be a decisive reason against it, especially if one has already abandoned certain commitments of standard realism, as proponents of OSR have. I will therefore now show that

this rather wide sense of "observation" in mind when I speak of "unobserved measurements" or "unobserved data". 
the proposed dissolution of the measurement problem conflicts not only with standard realism but also with elements of scientific realism that Ladyman and Ross themselves endorse.

A first hint of this conflict appears in the role that the notion of "data" plays within rainforest realism. As we saw in Section 2, Ladyman and Ross conceive of reality in terms of real patterns, and patterns are "relations among data" (2007, 228). In their discussion of Dennett's (1991) account of real patterns, Ladyman and Ross carefully distance themselves from the kind of instrumentalism that is at least partly invited by Dennett's writing and has preoccupied many of his commentators. In the process of doing so, they acknowledge that "there are (presumably) real patterns in lifeless parts of the universe that no actual observer will ever reach" (Ladyman and Ross 2007, 203). But such realism about patterns presupposes realism about data regardless of whether they are observed or not. The same kind of realism is required for the Peirce-inspired view of reality as "the totality of non-redundant statistics" that is developed in Ladyman and Ross (2013, Section 5) and will briefly be discussed in Subsection 5.2 below. This already shows quite clearly that Ladyman and Ross cannot accept the non-realism about unobserved quantum measurements that would follow from a strict verificationism with respect to QM.

The problem comes into sharper focus when we turn to Ladyman's and Ross's (2007, Subsection 2.3.2) critique of van Fraassen's constructive empiricism. While they largely share van Fraassen's aversion to traditional metaphysics, they defend a commitment to objective modality as a crucial element of realism against his deflationary view. One reason for this is that "theories are always modalized in the sense that they allow for a variety of different initial conditions or background assumptions rather than just the actual ones, and so describe counterfactual states of affairs" (110). A constructive empiricist might regard the claim that science gives us knowledge about non-actual states of affairs as unjustified, because all we ever experience is the actual. But this, according to Ladyman and Ross, neglects the fact that we can to some extent vary what becomes actual and still experience that our theories accurately predict what we observe. In other words, the empiricist relies on a somewhat arbitrary boundary when confining the content of our theories to a description of what actually occurs.

Insofar as this accurately describes the motivation for preferring OSR to constructive empiricism, an adherent of OSR should be equally dissatisfied with versions of $\mathrm{QM}$ which fail to give a non-anthropocentric account of measurement, because they involve a similar boundary between what our theories do and do not tell us. In this case, it is not the boundary between what actually occurred and what could have occurred under different initial conditions (if the Born rule is modalized in the above sense, it does give us knowledge about both of these), but the boundary between what was actually observed and what actually occurred without being observed (the Born rule being silent about the latter set of events). This second boundary is just as arbitrary as the first one, because it is largely up to us which occurrent events are observed and which ones are not.

The same point can also be made in terms of demands for explanation. In 
general, Ladyman and Ross share van Fraassen's s skepticism towards such demands, but here is one they explicitly accept: "That we are so often able to identify regularities in phenomena and then use them for prediction needs to be explained" (Ladyman and Ross 2007, 106). If OSR is to have any advantage over constructive empiricism, the sought-after explanation cannot simply be that there are such regularities, because that explanation would be available to the constructive empiricist as well. In order to satisfy OSR's demand, the regularities need to be invested with modal force, which enables us to answer questions about counterfactual situations. Among such questions are those about what would have happened if we had not been around to observe the phenomena in question, and an explanation would hardly be deemed satisfactory if it postulated regularities that only obtain if some observer is present. But this is precisely what the Born rule does, if it is interpreted as a modally charged law but not supplemented by a non-anthropocentric account of "measurement".

\section{Conclusion and remarks about related proposals}

I have argued (in Section 2 ) that neither the arguments for OSR or rainforest realism nor a commitment to naturalism serve to undermine the view that measurement devices are composed of quantum systems in the sense relevant for formulating the measurement problem. Furthermore, we saw that the proposal to dissolve the measurement problem along Bohrian lines conflicts not only with some commitments of standard realism (as demonstrated in Section 3 but also with the rather minimal kind of realism that Ladyman and Ross defend against van Fraassen (Section 4).

Some will object that this is very far from establishing the general claim contained in the title of this paper. Indeed, given the wide range of positions encompassed by the term "realism", it would be hopeless to deny that some proposed dissolutions of the measurement problem are compatible with some forms of realism. Let me nevertheless add a few remarks pointing towards a generalization of the above discussion. The two proposals I'm going to discuss have been suggested to me by an anonymous referee as possible interpretations of what Ladyman and Ross are aiming at. I will therefore not only examine these proposals with respect to their compatibility with realism but also ask whether they can legitimately be viewed as performing a similar task to the one that Ladyman and Ross have set for themselves.

\subsection{The pragmatist approach}

The first proposal is Richard Healey's (2017) pragmatist approach to QM. On the last pages of his book, Healey discusses the relation of his proposal to scientific realism and ultimately leaves it "to the reader to decide whether to classify the resulting view of quantum theory as realist as well as pragmatist" (257). In more recent work, however, he explicitly advertises his position as a version of realism (Healey forthcoming). The key idea is that QM, although it should not be viewed as a representation or description of the world, nevertheless 
gives us objective guidance for belief in certain magnitude claims, and these are the ones that truly describe (parts of) the world. Without going into further detail, let me just mention that one reason why a realist might not be satisfied with this approach is that it involves a rather peculiar contextualism about the content of magnitude claims (see Lewis forthcoming).

Be that as it may, what emerges quite explicitly from Healey's account is its incompatibility with the metaphysical project of Ladyman and Ross. Much of the support that QM lends to OSR depends on viewing quantum states (in particular, entangled states) as representing physical structure (although maybe not in the macroscopic realm, as we saw in Section 21, which is precisely what Healey denies. This leads him to conclude that "Quantum theory does not itself posit any novel physical structures so it does not make a contribution to fundamental physics, understood in Ladyman and Ross's way" (2017, 242). In other words, if Healey's approach is a kind of realism, then it is a realism that is even more minimal than the one defended by Ladyman and Ross. I thus suspect that it entails an even starker conflict with some standard realist and naturalist commitments than the one described in Section 3 , but I admit that more work is needed to assess the severity of this conflict.

\subsection{The information-theoretic interpretation}

The second proposal, Jeffrey Bub's (2016) information-theoretic interpretation of QM, is more interesting, because there is quite some prima facie evidence that it meshes very nicely with what Ladyman and Ross are doing. In addition to some approving comments on Bub's earlier work in Ladyman and Ross (2007, 184-186), they extensively rely on information theory in their articulation and defense of rainforest realism, culminating in what they call an "informationtheoretic conception of existence" $(2013,121)$. Hence, one might think that they would certainly join Bub in rejecting the "big" measurement problem (in the sense of Pitowsky 2006) as a pseudo-problem, which would leave us with only the "small" measurement problem of explaining how quantum probabilities get transformed into a classical probability distribution over macroscopic measurement outcomes.

I see two reasons for being suspicious of this connection. First, as Bub and Pitowsky (2010, 438) explain, rejecting the "big" measurement problem involves rejecting "two dogmas" about QM. While it is obvious that Ladyman and Ross do indeed reject the first dogma (namely, "that measurement should never be introduced as a primitive process in a fundamental mechanical theory"), this is not so clear with respect to the second dogma, "the view that the quantum state has an ontological significance analogous to the ontological significance of the classical state as the 'truthmaker' for propositions about the occurrence and non-occurrence of events, i.e., that the quantum state is a representation of physical reality". Although they might criticize talk of "truthmakers of propositions" (because it smacks of the syntactic view of theories, which they reject), we saw above that Ladyman and Ross are committed to a representational role of quantum states (as long as we do not apply them to macroscopic objects). The second reason for suspicion is that they do not seem to be concerned with 
anything that resembles Pitowsky's "small" measurement problem. The closest they get to discussing it is their account of decoherence mentioned in subsection 2.3 above, which, as we saw, takes place in the context of the Everettinterpretation, a view that $\mathrm{Bub}(2016,223)$ recognizes as a proposal to solve the "big" measurement problem.

But even if Ladyman and Ross could be interpreted as replacing the "big" measurement problem with the "small" one, this would do nothing to render the dissolution of the measurement problem acceptable to the realist. The resolute rejection of what Bub and Pitowsky call the "first dogma" still entails all the anti-realistic consequences discussed in Sections 3 and 4 . Furthermore, it is questionable whether Bub's realist rhetoric when he describes the solution of the "small" measurement problem is justified. He describes how "at the end of the measurement, the measured system $Q$ and the measuring instrument end up in a mixed state, which can be interpreted as a classical probability distribution over definite measurement outcomes" (227). These probabilities are "measures of ignorance over several possibilities, one of which actually happens" (225). Such an account faces an objection raised by Maudlin (1995, 9-10): True, when we are in a situation of ignorance concerning a distribution of classical possibilities, we can use a mixed state to describe the corresponding probabilities. But from this it does not follow that whenever a system is in a mixed state, there is something (namely, the real state) of which we are ignorant ${ }^{6}$

This is not to say that a realist account necessarily has to interpret probabilities in terms of ignorance. As Ladyman and Ross $(2013,146)$ point out, "dissatisfaction ... with irreducibly statistical fundamental physics ... has no sound motivation in either epistemology or naturalistic metaphysics". On the other hand, satisfaction with irreducible stochasticity does not entail satisfaction with a purely information-theoretic (as opposed to physical) analysis of "measurement". Adherents of spontaneous collapse models, for example, agree that reality is irreducibly stochastic, but deny that an information-theoretic understanding of the Born rule by itself provides us with a sufficiently realistic account of that stochasticity. In particular, they will ask for a physical description of the difference between information transmitting processes described by the Born rule and other such processes (in the microscopic realm) to which the Born rule apparently does not apply, as evidenced by the persistence of interference.

What this disagreement shows is that the issue of fundamental stochasticity is at least partly independent of the problems discussed in the previous sections. The information-theoretic realism captured in the slogan "the world is the totality of non-redundant statistics" (Ladyman and Ross 2013, Section 5) may offer a fascinating new framework for addressing questions of realism, but more

\footnotetext{
${ }^{6}$ If the proposal is amended by brutely postulating that one of the possibilities in the (classical) probability distribution gets selected, then (as Timpson (2010) observes) the view turns into a kind of modal interpretation that adds a value state to the usual quantum state. But since it then fails to say anything about how this state evolves, Timpson still deems it unacceptable to the realist.
} 
needs to be said about the conditions under which QM provides us with data about unobserved events. For without data, there is no statistics, and hence (according to the slogan) no world. In other words, a proper solution to the measurement problem will still be required for realism, even if it is couched in information-theoretic terms.

\section{Acknowledgments}

I would like to thank James Ladyman, Andrea Oldofredi and two anonymous referees for helpful comments in the development of this paper. I am also indebted to audiences in Leeds, Bern, Milan (SMS 2018) and Seattle (PSA 2018) for inspiring discussions.

\section{References}

Arndt, M. and K. Hornberger (2014). Testing the limits of quantum mechanical superpositions. Nature Physics 10, 271-277.

Bacciagaluppi, G. (2016). The role of decoherence in quantum mechanics. In E. N. Zalta (Ed.), The Stanford Encyclopedia of Philosophy (Fall 2016 ed.). Metaphysics Research Lab, Stanford University. https://plato.stanford. edu/archives/fall2016/entries/qm-decoherence/.

Bell, J. S. (1990). Against 'measurement'. In A. I. Miller (Ed.), Sixty-two years of uncertainty: historical, philosophical, and physical inquiries into the foundations of quantum mechanics, pp. 17-32. New York: Plenum Press.

Bub, J. (2016). Bananaworld: Quantum Mechanics for Primates. Oxford: Oxford University Press.

Bub, J. and I. Pitowsky (2010). Two dogmas about quantum mechanics. See Saunders et al. (2010), pp. 433-459.

Cordero, A. (2001). Realism and underdetermination: Some clues from the practices-up. Philosophy of Science 68, S301-S312.

Dennett, D. C. (1991). Real patterns. The Journal of Philosophy 88, 27-51.

Deutsch, D. (2011). The Beginning of Infinity. London: Allen Lane.

Egg, M. (2014). Scientific Realism in Particle Physics: A Causal Approach. Boston: De Gruyter.

Esfeld, M. (2013). Ontic structural realism and the interpretation of quantum mechanics. European Journal for Philosophy of Science 3, 19-32.

Healey, R. (2017). The Quantum Revolution in Philosophy. Oxford: Oxford University Press. 
Healey, R. (forthcoming). Pragmatist quantum realism. In S. French and J. Saatsi (Eds.), Scientific Realism and the Quantum. Oxford: Oxford University Press.

Ladyman, J. (2016). Are there individuals in physics, and if so, what are they? In A. Guay and T. Pradeu (Eds.), Individuals Across the Sciences, pp. 193206. Oxford: Oxford University Press.

Ladyman, J. (2017). An apology for naturalized metaphysics. In M. H. Slater and Z. Yudell (Eds.), Metaphysics and the Philosophy of Science: New Essays, pp. 141-161. New York: Oxford University Press.

Ladyman, J. and D. Ross (2007). Every Thing Must Go: Metaphysics Naturalized. Oxford: Oxford University Press.

Ladyman, J. and D. Ross (2013). The world in the data. In D. Ross, J. Ladyman, and H. Kincaid (Eds.), Scientific Metaphysics, pp. 108-150. Oxford: Oxford University Press.

Lewis, P. J. (forthcoming). Quantum mechanics and its (dis)contents. In S. French and J. Saatsi (Eds.), Scientific Realism and the Quantum. Oxford: Oxford University Press.

Maudlin, T. (1995). Three measurement problems. Topoi 14, 7-15.

Myrvold, W. (2017). Philosophical issues in quantum theory. In E. N. Zalta (Ed.), The Stanford Encyclopedia of Philosophy (Spring 2017 ed.). Metaphysics Research Lab, Stanford University. https://plato.stanford.edu/ archives/spr2017/entries/qt-issues/.

Pitowsky, I. (2006). Quantum mechanics as a theory of probability. In W. Demopoulos and I. Pitowsky (Eds.), Physical Theory and its Interpretation: Essays in Honor of Jeffrey Bub, Western Ontario Series in Philosophy of Science, pp. 213-239. Dordrecht: Springer.

Rosaler, J. (2016). Interpretation neutrality in the classical domain of quantum theory. Studies in History and Philosophy of Modern Physics 53, 54-72.

Saatsi, J. (forthcoming). Scientific realism meets metaphysics of quantum mechanics. In A. Cordero (Ed.), Philosophers Think About Quantum Theory. Springer.

Saunders, S., J. Barrett, A. Kent, and D. Wallace (Eds.) (2010). Many Worlds? Everett, Quantum Theory, and Reality. Oxford: Oxford University Press.

Timpson, C. (2010). Rabid dogma? comments on Bub and Pitowsky. See Saunders et al. (2010), pp. 460-466.

Wallace, D. (2003). Everett and structure. Studies in History and Philosophy of Modern Physics 34, 87-105. 